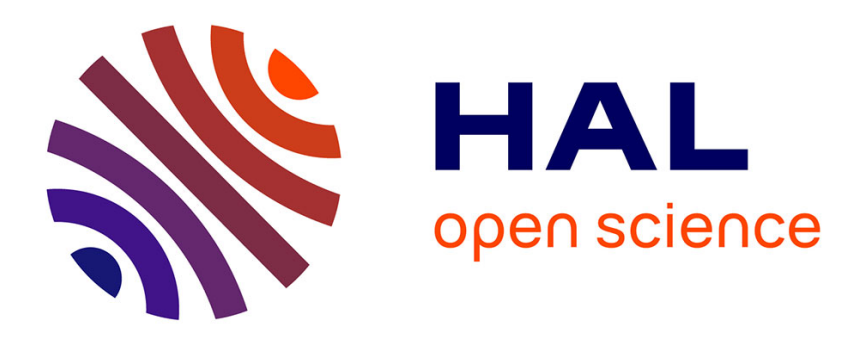

\title{
Universality of charge/spin density waves linear properties
}

S. Brazovskii

\section{To cite this version:}

S. Brazovskii. Universality of charge/spin density waves linear properties. Journal de Physique IV Proceedings, 1993, 03 (C2), pp.C2-267-C2-272. 10.1051/jp4:1993254 . jpa-00251337

\section{HAL Id: jpa-00251337 https://hal.science/jpa-00251337}

Submitted on 1 Jan 1993

HAL is a multi-disciplinary open access archive for the deposit and dissemination of scientific research documents, whether they are published or not. The documents may come from teaching and research institutions in France or abroad, or from public or private research centers.
L'archive ouverte pluridisciplinaire HAL, est destinée au dépôt et à la diffusion de documents scientifiques de niveau recherche, publiés ou non, émanant des établissements d'enseignement et de recherche français ou étrangers, des laboratoires publics ou privés. 


\title{
Universality of charge/spin density waves linear properties
}

\section{S. Brazovskii}

\section{Landau Institute for Theoretical Physics, Moscow, Russia}

\begin{abstract}
We reconsider microscopic grounds for the electric field and for the phase dynamics and relaxation of CDW/SDW, mostly for pure systems. We suggest a transparent form dynamic and dissipative equations for the DW phase and for electric field. The approach is based on a helpful relation between a "generalized condensate density" and a complex dielectric susceptibility of intrinsic carriers. Separately for CDW and SDW we discuss the spectra and the attenuation for the TO and LO modes, the low frequency relaxation, the intrinsic nonlinear conductivity.
\end{abstract}

\section{Introduction}

Recently we have suggested [1] a very general and transparent way to derive response and condensate properties of Density waves (DW). This approach helps to avoid special calculations of earlier studies by virtue of a manual book information on normal electron properties of a corresponding semiconductor or a semimetal. It is based on the observation that the total longitudinal force $F$ experienced by electrons under the DW phase deformation $\varphi=\varphi(x, t)$ and an applied electric field $E_{x}=E$ is given by the invariant combination $F=E-(v / 2) \varphi^{\prime \prime}+(1 / 2 v) \ddot{\varphi}$, where $v$ is the Fermi velocity of a parent metal (from now on the electron charge $e=1, \hbar=1$ ). The substitution of $E$ for $F$ corresponds to a transformation of electron wave function to a local frame of an arbitrary distorted DW phase. In this respect we distinguish between "extrinsic" and "intrinsic" carriers relative to the DW electronic spectral gap $\Delta$ vicinity in a sense that the first are subjected to the field $E$ solely while the second experience the combined force $F$. The responses of these particles $\epsilon_{e}$ and $\epsilon_{i}$ to $E$ and $F$ correspondingly are characterized completely by their partial complex dielectric function contributions $\epsilon_{a} ; a=e, i$ Relevant partial parameters $\lambda_{a}^{-1}$, $\omega_{a}$ and $\sigma_{a} / 4 \pi$ are the Debye screening lengths, the plasma frequencies and the conductivities of corresponding carriers. At $\Delta \rightarrow 0, \lambda_{a} \rightarrow \kappa=\omega_{p} / v ; \omega_{a}=\omega_{p}$ are the plasma frequency and the Debye length, which are parent metal parameters.

Surprisingly relations between $\varphi, E$ and electric induction $D$ and all their consequences can be written at all $0<T<T_{M F}$ and $\omega, v q_{\|}<\Delta$ in terms of $\epsilon_{i}$ and $\epsilon_{e}$ [1]. Neglecting extrinsic carriers as well as other host contributions we arrive at a specially simple form of these equations.

$$
\mathrm{a}: \quad \Omega^{2} \varphi=\left(\frac{\kappa^{2}}{\epsilon_{i}}-q^{2}\right)\left(\varphi+\frac{D}{\kappa^{2}}\right) ; \quad \text { b: }\left[\Omega^{2}-B q^{2}\right] \varphi=B E \quad \text { c: } \quad D=\epsilon E \quad(1 a, b, c)
$$

The total dielectric response $\epsilon$ in (1c) should be found by excluding $\varphi$ from (1a,b). In (1a,b)

$$
\text { a }: B(\vec{q}, \omega)=1-\epsilon_{i}(\vec{q}, \omega) q^{2} / \kappa^{2}, \quad \text { b: } \quad \Omega^{2}=\eta^{2}\left(\omega^{2} / u^{2}+i \gamma \omega-q_{0}^{2}-\alpha q_{\perp}^{2}\right) \quad(2 a, b)
$$


Here $q^{2}=q_{\|}^{2}-\omega^{2} / v^{2}, \eta=\Delta / \Delta_{0}, \vec{q}=\left(q_{\|}, q_{\perp}\right), \Delta=\Delta(T), \Delta_{0}=\Delta(0), \Delta\left(T_{M F}\right)=0 ; \Omega^{2}$ is a sum of restorting forces which are due to an interchain DW elasticity $\sim \alpha$, to a possible commensurability pinning and to the CDW lattice inertia; $u$ and $\gamma$ are lattice contribution to the CDW velocity and to a DW attenuation. Eq. (1b) shows that $B$ is the effective condensate density and the charge of the DW. Eq. (1a) relates it to the intrinsic carriers electric response $\epsilon_{i}$ which is the most instructive part of our approach. Typically we expect

$$
\epsilon_{i} \approx \epsilon_{\Delta}+\min \left\{\lambda_{i}^{2} / q_{\|}^{2},-\omega_{i}^{2} / \omega^{2}, i \sigma_{i} / \omega\right\} ; \quad \epsilon_{\Delta} \sim \omega_{p}^{2} / \Delta_{0} \Delta ; \quad \lambda_{i}^{2}=\kappa^{2} \rho_{n} ; \quad \omega_{i}^{2}=\omega_{p}^{2} \tilde{\rho}_{n}
$$

And $\tilde{\rho}_{n}<\rho_{n}<1$. Comparing (1a) and (3) we see that $\rho_{n}$ and $\rho_{s}=1-\rho_{n}(T)$ should coincide a priori with the BCS-like normal and condensate densities for a given $3 \mathrm{~d}$ electronic spectrum and nesting conditions. Analogously $\tilde{\rho}_{s}=1-\tilde{\rho}_{n}$ is the electronic contribution to the DW effective mass.Actually $B$ is not real, and taking it's imaginary part (dissipative screening) property into account is a source of disagreements between some our results and the earlier rather common ones.

In more details, near $T_{M F}$ we have a singular intraband contribution [2] at small $q_{\|}$and $\omega$. Following a standard narrow gap semiconductor formula for $\epsilon_{i}$ we find at $T>>v q_{\|}, \omega>>\tau_{i}^{-1}$ :

$$
\mathrm{a}: B \simeq \rho_{s}+\left(\tilde{\rho}_{s}-\rho_{s}\right) \sqrt{\frac{\omega^{2}}{\omega^{2}-\left(v q_{\|}\right)^{2}}} ; \quad \mathrm{b}: B\left(q_{\|}, 0\right)=\rho_{s} \sim \eta^{2} ; B(0, \omega)=\tilde{\rho}_{s} \sim \eta
$$

This is a transparent derivation for the two different $q_{\|}, \omega$ limits $f_{0} \propto \eta$ and $f_{1} \propto \eta^{2}$ [3] for the effective condensate density. Moreover we see that $B$ is real only at $\omega=0$ and at $\omega^{2}>v^{2} q_{\|}^{2}$, while the imaginary part dominates almost everywhere in the sector $q^{2}>0: \Im B / \Re B \sim(1 / \eta)|\omega / v q|>>$ 1 at $\eta<<\left|\omega / v q_{\|}\right|<1$. The second anomalous term in (4a) dominates everywhere when it is real at $q^{2}<0$. The singularity at $q \rightarrow 0$ in (4) saturates at $|v q / \omega|<\eta$ when $B$ reaches its parent metal limit $B=1$ so that $\mathrm{DW}$ is ignored near the zero sound resonance [3]. These effects may be viewed as a giant Landau damping of plasma modes enhanced here due to a negligible perpendicular dispersion of longitudinal velocity $v_{\|}$for an open Fermi surface. Unusually this velocity resonance affects also the real spectra. We should worn against interpretation of $B(\vec{q}, \omega)$ as an effective condensate density at $\omega \neq 0$ and especially against its separation near $T_{M F}$ in two asymptotic types of condensate densities: the static $f_{1} \propto \eta^{2}$ and the dynamic $f_{0} \propto \eta$ as in [3]. This concept may be misleading as in theory of [4] where the dynamic value $f_{0}$ was used in essentially thermodynamical calculations which can be hardly justified. Moreover for relatively low frequencies of sliding DW oscillations one certainly expects $\omega \tau_{i}<<1$ so that within the hypothesis $q_{\|}=0$ one would have $B=1-i \omega \sigma_{i} / \omega_{p}^{2} \simeq 1$ rather then $\eta$ or $\eta^{2}$. What is also discouraging in these interpretations is that as a function of $\omega B\left(\omega, q_{\|}\right)$is real only at these two limits $f_{1}$ at $\omega=0$ and $f_{0}$ at $\omega / q_{\|}=\infty$. In between the imaginary part dominates almost everywhere at $0<\omega^{2}<v^{2} q_{\|}^{2}$. This feature seems to have been overseen in previous studies where the relation (2) was not noticed and the singularity (4a) was not properly analyzed.

\section{The transverse optical spectrum.}

It is defined by the condition $E=0$ which we obtain from (1b) as the equation $B q^{2}=\Omega^{2}$. $C D W$ near $T_{M F}$. Following (2) and (4) the spectral equation acquires the form

$$
\omega^{2}\left(\frac{u^{2}}{v^{2}}+1\right)+i \omega \frac{\gamma}{v^{2}}+i \frac{C}{\eta} \frac{\omega}{v} \sqrt{q_{\|}^{2}-\frac{\omega^{2}}{v^{2}}}=q_{\|}^{2}+Q_{0}^{2} ; \quad Q_{0}^{2}=q_{0}^{2}+\alpha q_{\perp}^{2}
$$

We see that the bare DW self attenuation $\gamma$ acquires additional part $\gamma \Rightarrow \gamma+(C / \eta)(|q| / v), C \sim 1$. At $Q_{0}=0$ the attenuation may be relatively small and the TO spectrum can exist only if 
$\eta>>u / v$ i.e. far enough below $T_{M F}$ and provided that the mass enhancement is really large: $v / u \gg>1$. For $Q_{0} \neq 0$ there is a finite interval of nonattenuated oscillations $q_{\|}<Q_{0} u / v$ e.g. $\omega(0)=\tilde{u} Q_{0}, v^{2} / \tilde{u}^{2}=(v / u)^{2}+1 / \eta$.

The SDW at low temperatures. A remarkable feature of the SDW case $u \rightarrow \infty$ is that the regime $\Omega \approx 0$, may be achieved at $q_{\perp}=0$ neglecting own attenuation and the pinning. Then $\epsilon$ coincides with it's free electron form of the parent metal $\epsilon \Rightarrow \kappa^{2} / q^{2}$ at all temperatures [3]. If the SDW perturbations like $q_{\perp}, \gamma, q_{0}$ are considered then the intuitively expected SDW TA spectrum appears at $\omega^{2} \simeq v^{2} q_{\|}^{2}+Q_{0}^{2}, Q_{0}^{2}=q_{0}^{2}+\alpha q_{\perp}^{2}$. Notice that for $Q_{0}=0$ the $\epsilon_{i}$ contributions cancels at all $\omega, q_{\|}$, so that e.g. the intergap absorption at $\omega \geq 2 \Delta$ will not be visible unless a pinning or a perpendicular component of polarization provide the SDW restoring.

The $S D W$ near $T_{M F}$. At zero restorting force $Q_{0}=0$ there are no spectra except for the sound one $\omega=v q_{\|}$at all temperatures. But for $Q_{0} \neq 0$ the spectrum evolves differently then at low temperatures because the anomaleous term in (4) dominates being real unlike for the CDW case. In a very broad range of $\omega: 0<-q^{2}<<Q_{0}^{2}$ we find the spectral equation

$$
0<-q^{2}<<Q_{0}^{2} \text { i.e. }|\omega| / v>\left|q_{\|}\right|,|\omega| / v>>Q_{0} \eta: \quad \omega \sqrt{\omega^{2}-v^{2} q_{\|}^{2}}=v^{2} \eta Q_{0}^{2}
$$

so that e.g. the perpendicular dispersion curvature or the pinning frequency are renormalized as $\alpha \Rightarrow \alpha \eta, q_{0} \Rightarrow \eta^{1 / 2} q_{0}$. The spectrum (6) starts at the frequency $\omega(0)=v Q_{0} \eta^{1 / 2}$ at $q_{\|}=0$ and converges to Fermi velocity at large $q_{\|}$.

Very close to $T_{M F}$ at $\eta<<u / v$ a similar spectrum (6) exists also for the CDW case at $Q_{0} \neq 0$ when the attenuation can be avoided as long as $v\left|q_{\|}\right|<|\omega|$ holds for the solution of (4). Now the same equation (6) is applied but at more essential constraints to prevent crossing the dissipation borderline $\omega=v q_{\|}: v Q_{0} \eta<<|\omega|<<u Q_{0}$ i.e. at $Q_{0} \eta v / u<<|q|<<Q_{0}$.

\section{The longitudinal optical spectrum.}

It is defined by the condition $D=0$ which leads us from (1a) to any of the equivalent equations:

$$
\text { a : } B\left(\Omega^{2}+q^{2}\right)=\Omega^{2} \quad \text { or } \quad \text { b : } \quad \omega^{2}\left(\frac{\eta^{2}}{u^{2}}-\frac{1}{v^{2}}\right)-\frac{\kappa^{2}}{\epsilon_{i}}=q_{0}^{2}+\alpha q_{\perp}^{2}-q_{\|}^{2}
$$

Eq. (7b) demonstrates the sign inversion of both $\omega^{2}-$ and $q_{\|}^{2}-$ contributions. It will be responsible for the negative $q_{\|}$-dispersion of the CDW LO gap mode and for the absence of the gap mode for the SDW against and also for the CDW close to $T_{M F}$.

CDW LO spectra at low temperatures. At finite $q_{\|}^{2}>\rho_{n} \kappa^{2}$ the Coulomb gap at $\omega_{C} \sim \Delta u / v$ comes from (7b). But against commonly accepted expectations the $q_{\|}$dispersion is found with the opposite sign. This observation tells us that $\left(\right.$ at $\rho_{n}=0$ ) we deal with the top of a phonon spectrum rather than with the bottom of a heavy plasmon spectrum. The standard formulas [3] definitely give the positive sign of $q_{\|}^{2}$ corrections. This bare small positive curvature $\sim+q_{\|}^{2} / \kappa^{2}$ is majorated [1] by the large negative contribution $\sim-q_{\|}^{2} \xi_{0}^{2}$ from the $B$-factor in (7a). At the same time the perpendicular dispersion has a positive curvature so that in full $3 \mathrm{~d}$ picture the zero temperature "LO gap" is actually the saddle point of the phonon spectrum.

For finite $\lambda$ at small $q_{\|}$the Coulomb gap is screened which is usually supposed to lead to a sound spectrum $\omega \approx q_{\|} v_{L}, v_{L} \approx u / \sqrt{\rho_{n}}$. Actually the validity of linear spectrum is limited to rather high $T$ by a requirement of relatively high concentration of carriers when their plasma frequency $\omega_{i}$ is high enough to provide the static screening $\tilde{\rho}_{n}>(u / v)^{2}$ so that $v_{L}<\omega_{i} / \lambda_{i}=v_{T}$, where $v_{T}$ is the thermal velocity or a small Fermi velocity of remnant carriers, e.g. for $u / v \sim 10^{-1}$ we need $T>\Delta_{0} / 5$. Otherwise at lower $T$ when $(u / v)^{2}>\tilde{\rho}_{n}$ the Landau attenuation is known to become of the order of frequency and the LO spectrum disappears. The attenuation becomes weak 
again only at small $q_{\|}$and at finite $\omega$ when we come to the dynamic regime of normal carriers. Then for the CDW there are always two solutions:

$$
\mathrm{a}:(\omega / \tilde{u})^{2}=q_{0}^{2}+\alpha q_{\perp}^{2}-q_{\|}^{2}, \quad(v / \tilde{u})^{2}=(v / u)^{2}+\left(\tilde{\rho}_{s} / \tilde{\rho}_{n}\right) \quad \text { and } \quad \mathrm{b}: \quad \omega^{2}=\omega_{C}^{2}+\omega_{i}^{2} \quad(8 a, b)
$$

The low frequency $\omega_{1}$ is adjacent to the sound spectrum which existed for intermediate $T$. It describes either pinned $\left(q_{0} \neq 0\right)$ or nonaligned $\left(q_{\perp} \neq 0\right)$ modes. It is characterized by exponentially growing effective mass, to be compared to similar growing velocity at high $T$. Notice that the spectrum (8a) also has a saddle point structure so that it exists only at small $q_{\|}^{2}<\alpha q_{\perp}^{2}+q_{0}^{2}$. The low temperature regime have been missed in discussions of LO screened modes [2] while the spectral studies of high gap CDW $[5,6]$ may well fall to this region.

$S D W L O$ spectrum. For the SDW the linear spectrum due to static screening does not exist which follows formally from $(7 \mathrm{a})$ since in this case $\Omega^{2}<0$. There are no Coulomb gap modes below $\omega_{p}$ [3] since at $u \rightarrow \infty$ the $\sim \omega^{2}$ term in (7b) changes the sign [2]. But the low frequency regime (8a) which is due to negative $\epsilon_{a}$ exists for the $S D W$ for all $\rho_{n}<<1: \omega^{2}=\left(\tilde{\rho}_{n} / \tilde{\rho}_{s}\right) v_{T}^{2}\left(q_{0}^{2}+\alpha q_{\perp}^{2}-q_{\|}^{2}\right)$. $C D W$ and $S D W L O$ spectra near $T_{M F}$. As well as for the TO mode the LO spectrum exists strictly speaking only at $q^{2}<0$ when $B$ is real and positive. So it is defined at $\Omega^{2}<0$ which always holds for the SDW or at $\Omega^{2}>-q^{2}$ which is possible for the CDW. We find the two branches of the SDW LO spectra: naturally the high frequency one goes very close to the metal plasma spectrum and the low frequency one starts at the finite frequency $\omega(0) \approx v Q_{0} \sqrt{\eta}$ at $q_{\|}=0$ and approaches the Fermi velocity at $q_{\|}>>Q_{0} \sqrt{\eta}$. At all $q_{\|}$this branch goes very close to the TO spectrum (6).

For the CDW case similar results may hold but in a very constraint region $v q_{\|}<u Q_{0}$ when $\Omega^{2}<0$. At larger $q_{\|}$the lattice inertia reduces the ratio $\omega / q_{\|}$below $v$ i.e. to the region of strong attenuation. At relatively large $\eta>u / v$ the conventional low velocity LO mode appears from the overdamped region $\omega<v q_{\|}$. Again this LO mode is close to the low speed TO mode (5).

We must recognize some mistakes in describing LO modes in the earlier version [1a]:

\section{The LO mode attenuation and relaxation.}

Consider now in more details the frequency dispersion at $\vec{q}=0$ including the DW attenuation and the conductivity. For negligible attentation we find again the combined Coulomb gap and the normal plasma mode which corresponds to a high frequency solution of (7) at $q_{0}=q_{\perp}=q_{\|}=0$. At any arbitrary $T$ it is given as

$$
\omega_{L O}^{2}=\frac{\omega_{p}^{2}}{\epsilon_{\Delta}} \frac{\tilde{\rho}_{n}(\eta v / u)^{2}+\tilde{\rho}_{s}}{(\eta v / u)^{2}-1}
$$

This spectrum exists only for the CDW and not very close to $T_{M F}$ when the denominator is positive.

The low frequency LO relaxation $\Gamma_{0}$ is given by imaginary zeros of $\mathrm{Eq}$. (7) $\omega=i \Gamma_{\mathbf{0}}$. For various limits it simplifies as

$$
\Gamma_{0} \approx \frac{\sigma_{i}+\sigma_{D W}}{\epsilon_{\Delta}+\sigma_{i} \sigma_{D W} / \tilde{\omega}^{2}} \Rightarrow \begin{cases}\tilde{\omega}^{2}\left(\sigma_{i}^{-1}+\sigma_{D W}^{-1}\right), & \epsilon_{\Delta}<<\sigma_{i} \sigma_{D W} / \tilde{\omega}^{2} \\ \left(\sigma_{i}+\sigma_{D W}\right) / \epsilon_{\Delta}, & \epsilon_{\Delta}>>\sigma_{i} \sigma_{D W} / \tilde{\omega}^{2}\end{cases}
$$

where $\tilde{\omega}^{2}=\omega_{p}^{2}\left[(\eta v / u)^{2}+\tilde{\rho}_{s} / \tilde{\rho}_{n}\right]^{-1}$. The case (9a) corresponds to a well screened Coulomb gap when the dissipative part of $\epsilon_{i}$ dominates unlike the cases of the static and of the dynamic (8b) regimes of screening. Oppositely the case $(9 \mathrm{~b})$ corresponds to the nonscreened but overdamped Coulomb gap. In case (9a) the low frequency relaxation $\Gamma_{0}$ coexists generally with the oscillating mode while in case $(9 \mathrm{~b})$ there are only relaxational modes (two others are of higher rates then (9b)). 
The approximation (9a) corresponds to a frequently exploited result of [7] when, as we see here, one should imply lower bounds for the product of the normal and the DW conductivities. At intermediate $T<T_{M F}$ when $\eta \sim \rho_{s} \sim \rho_{n} \sim 1$ and $\sigma_{i} \sim \sigma_{m}$ (here $\sigma_{m}$ is a metallic conductivity at $T \geq T_{M F}$ ) the condition (9a) requires that for CDW: $\sqrt{\sigma D W \sigma_{m}}>10^{1} / O \mathrm{hm} \cdot \mathrm{cm}$; and for SDW: $\sqrt{\sigma_{D W} \sigma_{m}}>10^{5} / \mathrm{Ohm} \cdot \mathrm{cm}$. These condition may be satisfied only marginally even for the best conducting semimetalic CDW like in $\mathrm{NbSe}_{3}$ or for organic SDW where the giant $\epsilon_{\Delta}$ contribution competes with the very high conductivity.

For limiting cases $\eta \rightarrow 0$ and $\eta \rightarrow 1$ we should take into account a temperature dependence of $\tilde{\omega}$, via $\tilde{\rho}_{n} \sim \eta$ near $T_{M F}$ and especially the exponential dependence on $T$ of both $\tilde{\omega}$ and $\sigma_{i}$ via $\rho_{n} \sim \exp \left(-\Delta_{0} / T\right)$ at low $T$. In terms of scattering time $\tau_{i}=\sigma_{i} / \omega_{i}^{2}, \tau_{i}\left(T_{M F}\right)=\tau_{m}$ the same condition reads:

$$
T \rightarrow T_{M F}: \quad \frac{\sigma_{D W}(T)}{\sigma_{m}}>>\frac{1}{\Delta \tau_{m}^{2}\left[1+\eta(v / u)^{2}\right]} ; \quad T \rightarrow 0: \quad \frac{\sigma_{D W}(T)}{\sigma_{i}(T)}>>\frac{1}{\left[\Delta_{0} \tau_{i}(T)\right]^{2}}
$$

The denominators in (10) are large if $T$ is not very low since $\Delta_{0} \tau_{m}>>1$ by definition. Then there is a place for expected small ratios of $\sigma_{D W} / \sigma_{m}$ and consequently for the screened gap regime.

Oppositely in case of low conductivities $(9 \mathrm{~b})$ i.e at inequalities opposite to $(10)$ we find the overdamped Coulomb gap relaxation regime $(9 \mathrm{~b})$. All conductivities contribute additively to $\Gamma_{0}$ being reduced (tremendously for the SDW) by $\epsilon_{\Delta}^{-1}$. Nevertheless the LO relaxation is strong enough to suppress all oscillations: no gap mode can be seen in Raman or corresponding neutron scattering spectra.

\section{The applied field, the bias current and the nonlinear conductivity.}

Eq. (12a) describes completely local properties of the phase statics, dynamics and relaxation for a general nonlinear pinning problem except for dislocations vicinity when $\epsilon_{h}$ cannot be omitted [8]. It takes into account the countercurrents, screening and the Coulomb hardening (see [9] and references therein). The pinning force which is contained in the $\Omega^{2} \varphi$ term can be generalized as $q_{0}^{2} \varphi \Rightarrow \delta W_{p i n}(\varphi, \vec{r}) / \delta \varphi$, where $W_{p i n}$ is the pinning energy which is periodic in $\varphi$ and depends on coordinates $\vec{r}$ for the impurity case and $\omega, \vec{q} \Rightarrow(i \partial / \partial t,-i \partial / \partial \vec{r})$. The bias voltage $-E_{0} L$, or the total current $J$ are controlled by introducing the electrical induction $D$ of the whole sample. By definition we have in Fourier components, $D_{\omega}=\epsilon(\omega, 0) E_{0 \omega} ;-i \omega D_{\omega}=4 \pi J_{\omega}$. This approach contradicts in many respects to a typical view on the pinning problem based on Eq. (12b) where the local field $E$ is interpreted as a bias one $E_{0}$. (Studies [10], [11] in applications to CDW are among rare exceptions). First the Eq. (1a) shows clearly that the $D W$ driving force is not $E_{0}$ but a value $E^{*}$ determined by the total current $J$ :

$$
E^{*}=\left.\frac{D(\omega)}{\epsilon_{i}(\omega)}\right|_{\omega=0}=\frac{J}{\sigma_{i}}=\frac{J_{l n}+J_{s l}}{\sigma_{n}}=E_{0}+\frac{J_{s l}\left(E^{*}\right)}{\sigma_{n}} ; \quad J_{l n}=E_{0} \sigma_{n}, J_{s l}=-B \kappa^{2} \dot{\varphi}
$$

Here the relation was employed $J=E_{0} \sigma_{n}+J_{s l}$ which follows from Eqs. (12a,b) if we neglect the remnant distortion $\left\langle\varphi^{\prime \prime}\right\rangle$ which should and have been proved [12] to be present also in unpinned $\mathrm{DW}$ when $\dot{\varphi}$ is not large enough. We see that at $E_{0}<E_{T}$ when $J_{s l}=0, E^{*}=E_{0}$ so that the value of $E_{T}$ is given by $E_{0}$ directly. The model (12b) with $E \Rightarrow E_{0}$ may be applied to determine $E_{T}$ at $T \approx T_{M F}$, but at lower $T$ the Coulomb hardening $\rho_{s} \rightarrow \rho_{s} / \rho_{n}$ must be taken into account. It is ignored somehow in all applications to SDW in of the fact that at $T \sim 4 K \rho_{n} \sim 10^{-2}$.

At $E_{0}>E_{T}$ the $D W$ current is a function of $E^{*}$ rather than of $E_{0}$. We arrive at the following $I-V$ and differential characteristics

$$
\mathrm{a}: E_{0}=E^{*}-\frac{J_{s l}\left(E^{*}\right)}{\sigma_{n}} ; \quad \mathrm{b}: \sigma^{-1}=\frac{d E_{0}}{d J}=\frac{\sigma_{n}-\sigma_{s l}}{\sigma_{n}^{2}} ; \quad \mathrm{c}: \sigma_{s l}^{-1}=\frac{d E^{*}}{d J_{s l}\left(E^{*}\right)}=\sigma_{n l}^{-1}+\sigma_{n}^{-1}
$$


Here $J_{s l}\left(E^{*}\right)$ is a solution for the DW sliding problem (12a) under the force $E^{*}, \sigma_{n l}$ is the so called nonlinear conductivity which is usually obtained experimentally by differentiating the nonlinear current $J_{s l}=J-J_{n}$ over the voltage $E_{0}$. The proper information on the DW sliding is given by the dependence of $J_{s l}$ on $E^{*}$ and the inherent conductivity is the value $\sigma_{s l}$ rather than $\sigma_{n l}$. The corresponding resistance $\sigma_{s l}^{-1}$ is given as a sum of the nonlinear and of the normal resistances (12c). At small nonlinearity: $\sigma_{n} \gg \sigma_{n l}$ we have naturally $\sigma_{s l} \simeq \sigma_{n l}$. Otherwise the differential experimental data for $\sigma_{n l}$ must be recalculated following (12c) or current-voltage $J-V$ plots must be reexamined for $J_{n l}$ as a function of $J$ rather then of $E_{0},(12 \mathrm{a})$. In the absence of pinning

$$
\mathrm{a}: \quad \sigma_{n l} \Rightarrow \sigma_{D W}=\frac{\kappa^{2}}{\gamma} ; \quad \mathrm{b}: \quad \sigma_{s l}^{-1} \Rightarrow \sigma_{D W}^{-1}+\sigma_{n}^{-1} ; \quad \text { c: } \quad \sigma_{s l} \approx \frac{\tilde{\omega}^{2}}{\Gamma_{0}} \quad(13 a, b, c)
$$

The approximate relation (13c) corresponds to the case (9a). Eq. (12a) provides also [1] a natural phenomenological classification of $J-V$ curves including the bistability effect of [10] and other possibilities.

\section{Conclusions}

Near $T_{M F}$ above single particle attenuation rate both LO and TO spectra are dominated by singularities of $\epsilon_{i}$ at the Fermi velocity line $q^{2}=q_{\|}^{2}-\omega^{2} / v^{2}=0$. For the CDW the conventional low velocity mode falls into the strong Landau damping region. At lower $T$ the existence of a screened LO sound mode is confirmed only at rather high concentration of normal carriers otherwise the static screening with growing velocity changes to a dynamic one with growing effective mass.

The relaxation rate $\Gamma_{0}$ for microscopic distortions of $E$ and $\varphi$ is found to be linear in partial resistivities (9a) or in conductivities (9b) depending on the product of the DW conductivity and of the normal one which falls just to experimental regions of parameters.

For an unpinned sliding $\mathrm{DW}$ we find the driving force $E^{*}$ to be determined by the total current as $E^{*}=J / \sigma_{n}$ rather then by the bias voltage strength $E_{0}$ which is different from $E^{*}$ above $E_{T}$. This observation suggests that the usual procedure to determine the inherent DW conductivity must be reconsidered. It explains phenomenologically the mixing of the DW and the normal conductivities and the possible $I-V$ instabilities [1].

Acknowledgements. The author acknowledges the hospitality of the Laue-Langevin Institute and the CNRS support during his stay in France where this work was performed. The assistance by N. Kirova was invaluable in preparing the manuscript.

\section{References}

[1] Brazovskii S.A., a: Preprint ILL, February 1993, b: J. Phys. I, France, to be published.

[2] Lee P.A., Rice T.M., Gross M.C., Phys. Rev. B20 (1979) 1345.

[3] Virosztek A., Maki K., These Proceedings.

[4] Maher M., Ramakrishna S. et all, These Proceedings.

[5] Hennion B., Pouget J.P., Sato M., These proceedings.

[6] Currat R., Lorenzo J.E., Monceau P., Hennoin B., Levy S., These Proceedings.

[7] Littlewood P.B., Phys. Rev. B36 (1987) 3108.

[8] Brazovskii S.A., These Proceedings.

[9] Richard J., Chen J., Artemenko S.N., These Proceedings.

[10] Littlewood P.B., Solid State Commun. 65 (1988) 1347.

[11] Wonneberger W., Synth. Met. 43 (1991) 3793.

[12] Brock J.D., These Proceedings. 